\title{
ATUAÇÃO DE UMA EQUIPE MULTIPROFISSIONAL NA ASSISTÊNCIA PRÉ- NATAL E PUERPERAL: UM RELATO DE EXPERIÊNCIA
}

\author{
MULTIPROFESSIONAL TEAM PRENATAL AND PUERPERAL CARE \\ PERFORMANCE: AN EXPERIENCE REPORT
}

\author{
Renata Cristina Alves, Jessica Vertuan Rufino, Thais da Silva Capello, Anna Luisa Gobbo \\ Catharino, Fabiana Fontana Medeiros
}

Universidade Estadual de Londrina - UEL

\begin{abstract}
The "Programa de Residência Multiprofissional em Saúde da Mulher" (Women's Health Multiprofessional Residency Program) was created as a strategy to improve the integral assistance of women's health, with gestation and puerperiumn being one of the most focused upon phases. In order to build a transdisciplinary vision of women's health assistance, this paper aims to report on the experience of a multiprofessional team in shared prenatal and puerperal care. The used methodology consists in performing prenatal appointments and puerperal home check-ups executed by the team in a Basic Health Unit in the city of Londrina-PR. This experience enriches the professionals trough information exchange and consequently, professional growth.
\end{abstract}

Key words: Prenatal care. Puerperiumn. Women's health.

\section{Resumo}

O Programa de Residência Multiprofissional em Saúde da Mulher (RMSM) foi criado como estratégia para melhoria da assistência integral à saúde da mulher, sendo a gestação e puerpério uma das fases de maior enfoque. Tendo em vista a construção de uma visão transdisciplinar da assistência à saúde da mulher, o presente trabalho tem como objetivo relatar a experiência de uma equipe multiprofissional na assistência ao pré-natal e puerpério compartilhados. A metodologia empregada consiste na realização de consultas de pré-natal e visitas puerperais pela equipe em uma Unidade Básica de Saúde no município de Londrina-PR. Essa experiência traz aos profissionais um enriquecimento pelo fato de haver troca de informações e consequentemente, crescimento profissional.

Palavras chaves: Assistência pré-natal. Puerpério. Saúde da mulher. 
Introdução

O Ministério da Saúde (MS) vem apoiando Residências Multiprofissionais em Saúde (RMS) desde 2002, tendo como objetivo possibilitar tanto a formação de profissionais quanto contribuir com a mudança do desenho tecnoassistencial do Sistema Único de Saúde (SUS). Visto que o SUS têm requerido a reformulação da atuação dos diversos atores que compõem o quadro da atenção à saúde, as equipes multiprofissionais surgem como uma proposta de assistência baseadas nos princípios da universalidade, integralidade, equidade, intersetorialidade, humanização do atendimento e participação social propostas pelo SUS ${ }^{1}$.

Dentro da proposta de olhar para as populações mais vulneráveis, respeitando os princípios do SUS, surge o Programa de Residência Multiprofissional em Saúde da Mulher (RMSM), financiado pelo MS e vinculado à Universidade Estadual de Londrina (UEL) - PR. O programa está inserido como estratégia para melhoria da assistência integral à saúde da mulher nos três níveis de atenção do SUS, compreendendo ações de promoção, proteção, assistência e recuperação da saúde, voltadas às mulheres em todos os ciclos de vida, sendo a fase da gestação e puerpério uma das áreas de maior enfoque do programa.

As mulheres vêm conquistando um espaço cada vez maior no mundo, o movimento atual de transformações sociais e econômicas tem contribuído para um cenário de mudanças tanto nas relações de trabalho como na inserção social das mulheres. Compondo a maior parte da população brasileira, são as principais usuárias do SUS, utilizando os serviços para o seu próprio atendimento e, como acompanhantes ${ }^{2}$.

Durante a vida, a mulher passa por ciclos, os quais necessitam de cuidados diferencias e um olhar especial da equipe de saúde. Um dos ciclos que exige maior atenção é o ciclo gravídicopuerperal, no qual a mulher apresenta alterações biológicas, fisiológicas e sociais ${ }^{3-4}$. Sendo assim, desde o início do século XX foram implementadas políticas nacionais de saúde voltadas ao acompanhamento materno-infantil, por se tratarem de grupos vulneráveis ${ }^{5}$.

Tendo em vista a construção de uma visão transdisciplinar da assistência à saúde da mulher, visando o cuidado integral na atenção primária, o presente trabalho tem como objetivo relatar a experiência de uma equipe multiprofissional na assistência ao pré-natal e puerpério compartilhados.

\section{Metodologia} Saúde

O Programa de Residência Multiprofissional em

O Programa de Residência Multiprofissional em Saúde caracteriza-se por um ensino de pósgraduação lato sensu que tem como objetivo a especialização por meio do ensino na prática do SUS, sob supervisão de outros profissionais vinculados ao serviço. O curso tem duração de dois anos, com carga horária de 60 horas semanais, totalizando 5.760 horas, sendo $20 \%$ carga horária teórico e teórico-prática e $80 \%$ carga horária prática ${ }^{6}$.

As profissões abrangidas pelo programa são: Biomedicina, Ciências Biológicas, Educação Física, Enfermagem, Farmácia, Fisioterapia, Fonoaudiologia, Medicina Veterinária, Nutrição, Odontologia, Psicologia, Serviço Social e Terapia Ocupacional ${ }^{7}$.

Os programas em saúde apresentam grandes variedades entre si, porém todos possuem o mesmo objetivo, que é a capacitação de profissionais para o SUS. A criação de uma equipe multidisciplinar tende a romper o modelo de saúde assistencialista e biologicista, visando o atendimento de forma integral, respeitando as diretrizes e princípios propostos pelo SUS ${ }^{1}$.

\section{O nosso Programa de Residência}

O Programa de Residência Multiprofissional em Saúde da Mulher foi proposto pelo Departamento de Enfermagem da UEL em 2011, com aprovação finalizada em dezembro de 2012 e concessão de bolsas de estudo financiadas pelo Ministério da Saúde, contemplando cinco áreas profissionais, sendo oito vagas para enfermagem, duas para psicologia, duas para nutrição, duas para farmácia e duas para profissionais de educação física.

Durante o primeiro ano da residência os profissionais são alocados em Unidades Básicas de Saúde (UBSs), sendo divididos em quatro equipes, duas compostas por: dois enfermeiros, um nutricionista e um farmacêutico; e duas por: dois enfermeiros, um psicólogo e um profissional de educação física. Já no segundo ano, os residentes fazem rodízio entre diferentes campos de prática, abrangendo atenção secundária e terciária.

A especialização é caracterizada por treinamento em serviços, tendo como orientação os professores das respectivas áreas do corpo docente da UEL, sendo denominados tutores. As atividades são desenvolvidas com articulação 
entre ensino, serviço e comunidade, uma experiência enriquecedora para todos os envolvidos. No campo de prática os residentes são coordenados por um profissional denominado preceptor.

O objetivo da RMSM é formar profissionais com visão multi e interdisciplinar para promoção, prevenção e recuperação da saúde da mulher em idade reprodutiva (10 a 59 anos), nos diferentes níveis de atenção, afim de melhorar a qualidade de vida da mulher.

\section{Atuação Multiprofissional no Pré-Natal e Puerpério}

A assistência à saúde da mulher no período gravídico-puerperal é de extrema importância visto que a redução dos óbitos materno-infantil, se relacionam com a melhora da qualidade de vida e assistência dentro de um País. O acompanhamento no pré-natal tem como objetivo assegurar um bom desenvolvimento do binômio mãe e filho, abordando inclusive aspectos psicossociais, atividades educativas e preventivas $^{8}$.

Considerando a importância da identificação de fatores de risco relacionados com a mortalidade materno-infantil, em 2012 a Secretaria de Estado do Paraná implantou a Rede Mãe Paranaense para orientar o planejamento das ações e redução desses indicadores. A partir de então, para melhor assistência à gestante e suas necessidades, o programa criou critérios para a estratificação do risco gestacional, sendo este classificado em: risco habitual, intermediário e alto risco ${ }^{9}$. 0 puerpério imediato ocorre nos primeiros dias pós-parto com ações básicas preconizadas pelo $\mathrm{MS}$, que objetiva a triagem neonatal, auditiva, checagem de vacinação e avaliação do aleitamento materno, são dadas orientações e o apoio quando necessário ${ }^{8}$.

Devido a importância da realização do prénatal, puerpério e o cuidado integral da mulher, foi desenvolvido este relato de experiência pelas residentes do primeiro ano da RMSM. A equipe é composta por duas enfermeiras, uma nutricionista e uma farmacêutica alocadas em uma Unidade Básica de Saúde na zona leste do município de Londrina-PR.

São realizadas consultas de pré-natal e visitas puerperais pela equipe, nas quais são acompanhadas gestantes independente do risco e idade gestacional. Nas consultas compartilhadas, são realizados atendimentos pelas enfermeiras, que consistem na verificação de sinais vitais, exame físico, solicitação e avaliação de exames laboratoriais; pela nutricionista, evolução do estado nutricional e realização do Recordatório Alimentar de 24h; e pela farmacêutica, abordando à adesão da suplementação de sulfato ferroso e ácido fólico e cuidados com a automedicação.

As visitas puerperais são realizadas entre sete e dez dias pós-parto, conforme preconizado pelo $\mathrm{MS}^{8}$. As visitas compartilhadas aconteciam juntamente com o agente comunitário de saúde (ACS) da área e quando possível com a técnica em saúde bucal (TSB). São abordados temas como cuidados no pós parto, alimentação, aleitamento materno exclusivo, planejamento familiar, uso do sulfato ferroso, cuidados com a automedicação e com o recém-nascido (RN), além da observação do ambiente domiciliar, vínculo entre mãe e filho e da família com o binômio.

Em alguns casos não é possível realizar as visitas no período preconizado devido a dificuldades ao entrar em contato com a puérpera. Sendo assim era realizada busca ativa pelo ACS da área, o qual solicitava o seu comparecimento até à UBS para realização desta consulta. Em outras situações as pacientes se encontravam fora da área de abrangência da unidade, devido à mudança de endereço.

\section{Conclusão}

Durante este primeiro ano da residência foi possível vivenciar a importância da equipe multiprofissional na resolutividade da demanda encontrada na UBS, visto que cada profissional pode contribuir com o seu conhecimento específico, visando a assistência integral ao paciente.

Essa experiência traz aos profissionais um enriquecimento pelo fato de haver troca de informações e consequentemente, crescimento profissional. A equipe trabalha para desenvolver habilidades que permitam atendimento de qualidade, objetivando o acolhimento, acompanhamento, orientações, busca ativa e encaminhamentos necessários aos demais profissionais que atuam no cuidado direto na unidade básica de saúde e níveis complementares.

\section{Referências}

1. Brasil. Ministério da Saúde. Secretaria de Gestão do Trabalho e da Educação na Saúde. Departamento de Gestão da Educação na Saúde. Residência multiprofissional em saúde: experiências, avanços e desafios / Ministério da Saúde, Secretaria de Gestão do Trabalho e da 
Educação na Saúde, Departamento de Gestão da Educação em Saúde. - Brasília: Ministério da Saúde, 2006.

2. Brasil. Ministério da Saúde. Secretaria de Atenção à Saúde. Departamento de Ações Programáticas Estratégicas. Política Nacional de Atenção Integral à Saúde da Mulher: Princípios e Diretrizes / Ministério da Saúde, Secretaria de Atenção à Saúde, Departamento de Ações Programáticas Estratégicas. - 1. ed., 2. reimpr. Brasília: Ministério da Saúde, 2011.

3. Costa ES, Pinon GMB, Costa TS, Santos RCA, Nóbrega AR, Sousa LB. Alterações fisiológicas na percepção de mulheres durante a gestação. Rev. Rene. Fortaleza, v. 11, n. 2, p. 8693.2010.

4. Cardoso JP, Pires AP. Perturbações do comportamento alimentar na gravidez: uma revisão. Psicol. Reflex. Crit. [online]. 2012, v. 25, n.1, pp. 139-146. Disponível em: <http://dx.doi.org/10.1590/S0102-

79722012000100017>. Acesso em: 16 de janeiro de 2017.

5. Brasil. Ministério da Saúde. Secretaria de Atenção à Saúde. Departamento de Ações Programáticas Estratégicas. Política nacional de atenção integral à saúde da mulher: princípios e diretrizes. Brasília: Editora do Ministério da Saúde, 2009. 83p.

6. Brasil. Ministério da Educação. Altera o art. 10- da Portaria Interministerial no45/ME/MS, de 12 de janeiro de 2007, que dispõe sobre a Residência Multiprofissional em Saúde e a Residência em Área Profissional da Saúde. Portaria Interministerial n. 506, de 24 de abril de 2008. Distrito Federal: Dou, 25 abr. 2008. Disponível em: <http://portal.mec.gov.br/sesu/arquivos/pdf/resi dencia/portaria_506_08.pdf>. Acesso em: 26 jan. 2017.

7. Brasil. Ministério da Educação. Dispõe sobre a Residência Multiprofissional em Saúde e a Residência em Área Profissional da Saúde e institui a Comissão Nacional de Residência Multiprofissional em Saúde. Portaria Interministerial n. 45, de 12 de janeiro de 2007. Distrito Federal: Dou, 15 jan. 2007. Disponível em:

<http://portal.mec.gov.br/sesu/arquivos/pdf/resi dencia/portaria_45_2007.pdf>. Acesso em: 26 jan. 2017.

8. Brasil. Ministério da Saúde. Secretaria de Atenção à Saúde. Departamento de Atenção Básica. Atenção ao pré-natal de baixo risco [recurso eletrônico] / Ministério da Saúde. Secretaria de Atenção à Saúde. Departamento de Atenção Básica. - 1. ed. rev. - Brasília: Editora do
Ministério da Saúde, 2013. 318 p.: il. - (Cadernos de Atenção Básica, n 32).

9. Paraná. Secretaria de Estado de Saúde do Paraná. Linha Guia: Rede Mãe Paranaense. 6 de outubro de 2014.

\section{Endereço para Correspondência}

Rodovia Celso Garcia Cid - Pr 445 Km 380 Cx. Postal 10.011

Campus Universitário, PR

CEP.: 86057-970

e-mail: renata7b@gmail.com

Recebido em 03/10/2017

Aprovado em 04/04/2018

Publicado em 30/09/2018 